\title{
VII.
}

\section{Ueber die Histogenese und das Wachsthum des Carcinoms.}

\author{
Von Prof. Dr. Hugo Ribbert \\ in Zürich.
}

(Hierzu Taf. V.)

Im 135. Bande dieses Archivs habe ich über Untersuchungen zur Histogenese des Carcinoms berichtet. Ich gelangte dabei zu Resultaten, die ich in dem Schlussabschnitt meiner Arbeit folgendermaassen zusammenfasste:

„Die Entwickelung eines Carcinoms der äusseren Haut wird eingeleitet durch lebhafte Proliferationsprozesse im Bindegewebe, welche zur Bildung einer subepithelialen, mehr oder weniger dicken Schicht „zelliger Infiltration" führen. Gleichzeitig erfolgt eine Verdickung des darüber gelegenen Epithels, welches aber zunächst noch keine Wachsthumserscheinungen zeigt, die zu einem Ueberschreiten seiner unteren Grenze führen. Die Verlängerung der Epithelzapfen beruht auf der in Folge der bindegewebigen Wucherungsprozesse eintretenden Erhöhung der Papillen. Die Metastasirung des Epithels in das Bindegewebe kommt nicht durch sein Vordringen in die Tiefe zu Stande, sondern dadurch, dass die Bindegewebszellen in die Epithelzapfen hineinwandern und hineinwuchern und die Zellen derselben auseinanderdrängen und isoliren. Von den so zwischen die Bestandtheile des neugebildeten Bindegewebes gelangten Epithelzellen geht die eigentliche Krebsentwickelung aus, indem sie Alveolen bilden und strangförmig tiefer in das Bindegewebe hineinwachsen. Dabei ordnen sie sich entweder völlig geschlossen an oder so, dass sie einzeln neben und hinter einander in die Spalten der Bindesubstanz vordringen und die Bestandtheile derselben, Fasern und Zellen, zwischen sich einschliessen. Vermehren sie sich dann in solchen Abschnitten weiter, so können sie abgegrenzte 


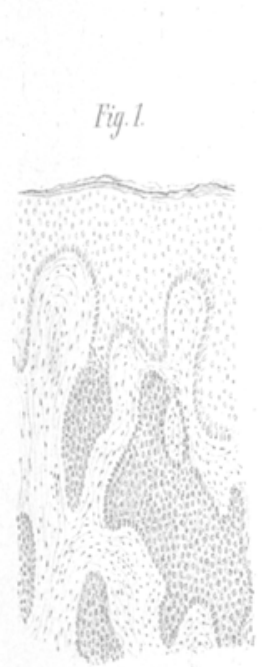

Fig. 3.

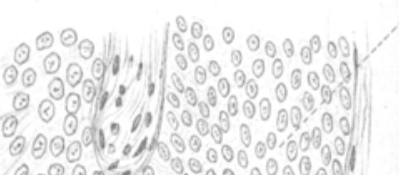
0.00000000000
0.000
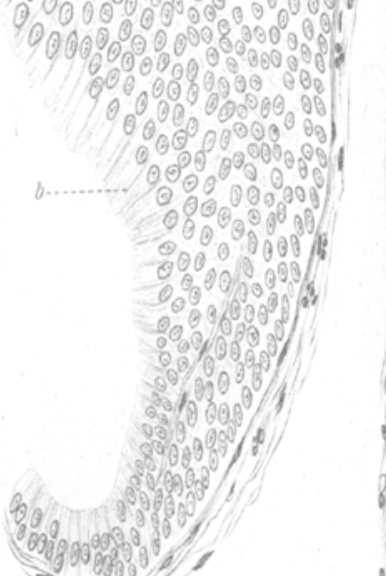

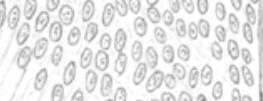

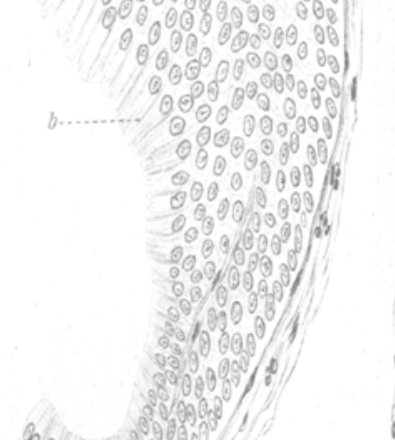

Fig. 2.
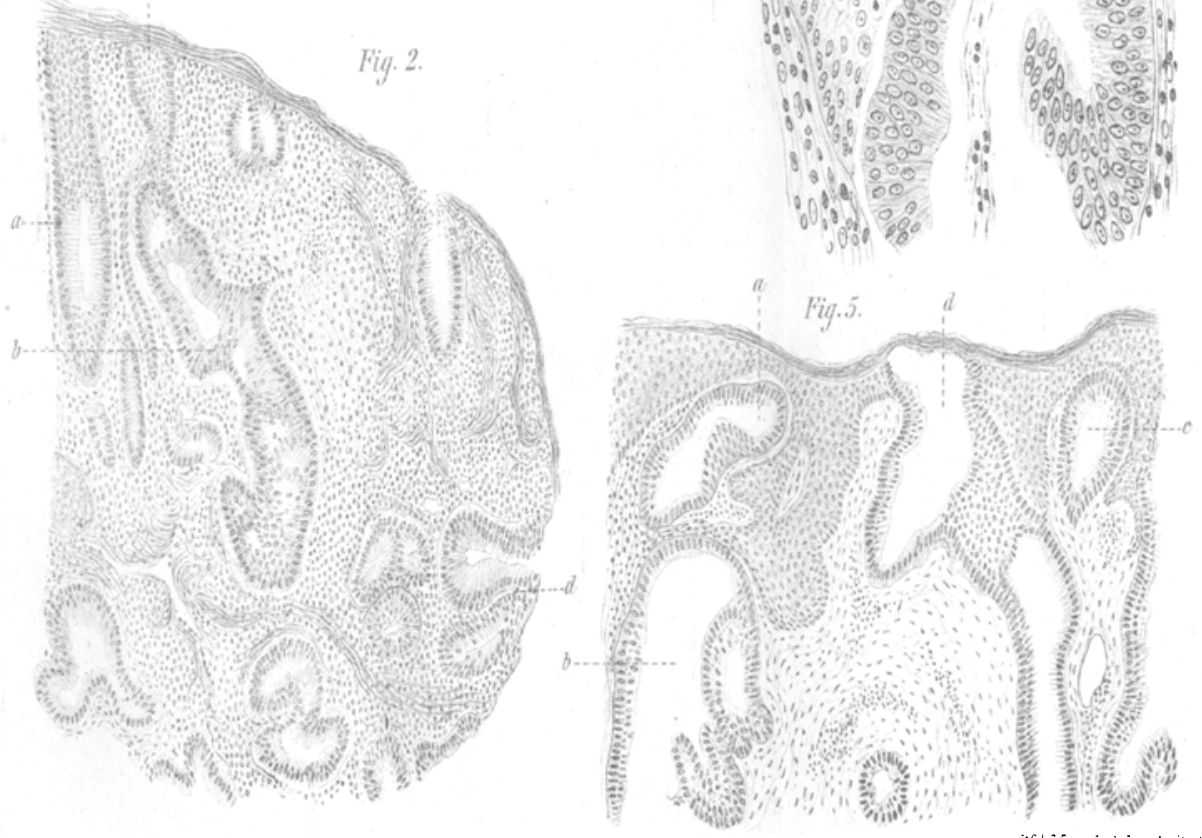

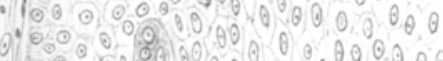

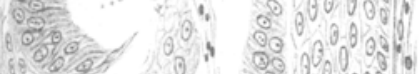

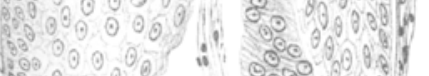

in $80 \%$

00,0200

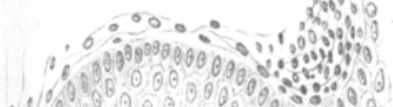

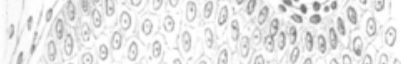

$1030 \% 00000000000000$

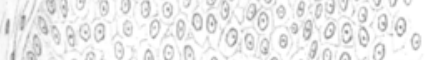

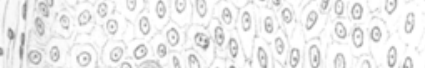

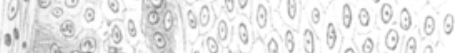

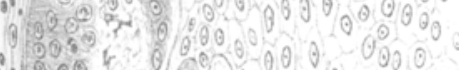

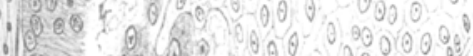

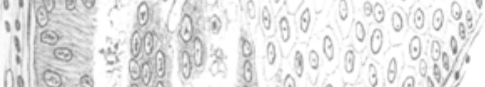

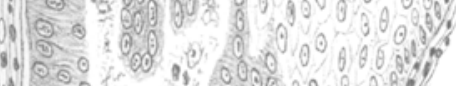

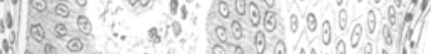

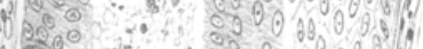

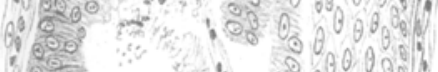

10 (8)

1800\%

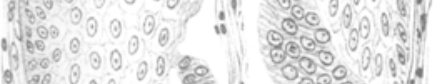

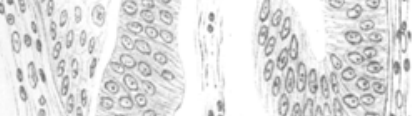

1,94800

$8_{3} \cdot \frac{000}{0.00}$

oromisos:

000050

$0.09 \%$ 
Alveolen bilden, in denon aber zwischen den wiphellen noch die eingeschlossenen Theiles des Bindegewebes wahrnehmbar bleiben."

Kurz darauf habe ${ }^{1}$ ) ich diese zunächst nur durch die Untersuchung von Hautcarcinomen gewonnene Vorstellung auch auf die Schleimhautkrebse übertragen und zwar an der Hand eines kleinen, eben beginnenden Nagencarcinoms. Der Tumor zeigte eine erbebliche Verdickung der Mucosa durch zellige Infiltration des Bindegewebes und Verlängerung der Drüsen, also analoge Befunde, wie bei den Krebsen der Haut. An vielen Stellen fand sich ferner eine Zerlegung der Drüsen in einzelne Theile und isolirte Zellen, die in dieser Form in das zellreiche Bindegewebe verlagert waren. Auch diese Verhältnisse entsprechen den an der Haut beschriebenen. Der krebsige Charakter des Prozesses ging nun daraus hervor, dass im Bereich der durch die Zertheilung der Drüsen ausgezeichneten Abschnitte Gruppen und Haufen yon Epithelien die Muscularis mucosae durchbrachen und unterhalb derselben, aber nur in den obersten zellig infiltrirten Schichten der Submucosa und nur in relativ geringem Umfange weiterwuchsen. Auch hier schloss ich, dass die aus dem Verbande der Drüsen gelösten und isolirten Zellen für sich proliferirend und den Lymphbahnen folgend das Carcinom erzeugten.

Diese Vorstellungen äber die Histogenese der Krebse habe ich forner noch einmal kurz besprochen in einem Artikel uber die Entstehung der Geschwülste ${ }^{2}$ ) überhaupt, in welchem ich darzulegen versuchte, dass die Bildung der Tumoren zurückgeführt werden kann auf eine intrauterin oder extrauterin erfolgende Trennung von Gewebskeimen aus dem Zusammenhang. Die isolirten Zellgruppen, die dem Einfluss des organischen Ganzen entzogen sind, wachsen nun für sich und führen deshalb zu mehr oder weniger atypischen, unregelmässigen Neubildungen, aben den Geschwälsten. Bei dem Carcinom wird die Isolirung der Epithelien durch die Bindegewebswucherung bedingt.

Es konnte nun nicht ausbleiben, dass meine Darlegungen über die Carcinomentwickelung Widerspruch erfahren würden.

1) Centralbl. für patholog. Anat. 1894. S. 697.

b) Deutsche med. Wochensehr. 1895. No.1-4. 
Standen sie doch in ausgesprochenen Gegensatz zu Allem, was man bis dahin über diesen Gegenstand annahm. Niemand hatte ernstlich daran gezweifelt, dass das Epithel aus sich heraus in das Bindegewebe vordränge und zur Stütze dieser Anschaunng hatte man verschiedene Theorien aufgestellt. Ausführlich ist mir indessen bis jetzt nur Ha user ${ }^{1}$ ) entgegengetreten, indem er sich auf Grund seiner bekannten werthvollen Untersuchungen über das Cylinderepithelcarcinom vor Allem gegen meine Darstellung von der Genese des Magenkrebses wandte, dabei aber auch die allgemeineren Gesichtspunkte der Krebsentwickelung berücksichtigte. Im Uebrigen habe ich in der Literatur nur einige ganz kurze absprechende Urtheile gefunden, die mir zu Bemerkungen keine Veranlassung geben.

Wenn ich nunmehr nochmals auf die Histogenese des Carcinoms eingehe, so liegt mir daran, die Gründe für meine Ansicht zu verstärken und die erhobenen Einwände zu entkräften. Ich will das in der Weise thun, dass ich die einzelnen in Betracht kommenden Punkte der Reihe nach durchgehe und die Besprechung der dagegen geltend gemachten Bedenken daran anknüpfe.

Im Gegensatz zu meinen Darlegungen kam Hauser zu dem Schluss, dass die Krebswucherung vom Epithel ausgehe, welches fähig werde, in das Bindegewebe vorzudringen, Den Grund dieses abnormen Wachsthumsvermögens suchte er mit Hansemann in der von diesem betonten Entdifferencirung, der „Ana-

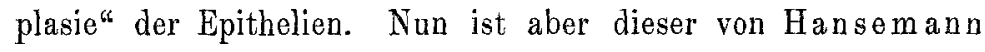
in seiner Monographie „Studien über die Specificität, den Altruismus und die Anaplasie der Zellen" auf Grund von vortrefflich durchdacbten Ueberlegungen aufgestellte Begriff lediglich abstrahirt aus gewissen morphologischen Verhältnissen, unter denen das häufige Vorkommen asymmetrischer Kerntheilungsfiguren das Wichtigste war. Ich verzichte des Raumes wegens darauf, den Gedankengang Hansemann's über die Bedeutung dieser Gebilde hier zu reproduciren. Aber seitdem wir nun wissen, dass die asymmetrischen Mitosen nichts für das Carcinom Charakteristisches darstellen, ist den Ausführungen Hansemann's die Stütze entzogen, welcher allein einige Bedeutung zukam. Denn

1) Dieses Archiv. Bd. 138, S. 482 。 
die sonstigen auffallenden Befunde an den Zellen un Kernen Iassen sich ebeaso wie abnorme Kerntheilungsprozesse lediglich als secundäre, von den verïnderten Wachsthumsbedingungen abängige Erscheinungen erklären. Wäre dem nicht so, so sollte man jene Dinge, da sie doch die Grundlage der Krebsentwickelung darstellen würden, in erster Linie auch in den beginnenden Tumoren finden. Aber gerade hier vermisst man sie nach meiner Erfahrung voollig, oder findet sie wenigstens nicht reichlioher, als in einfachen, epithelialen Hyperplasien.

Wenn sich nun charakteristische anatomische Eigenthümlichkeiten an den Krebszellen nicht feststellen lassen, so kann sich die Vorstellung von einer biologischen Aenderung der Epithelzellen, durch welche sie zu einem Vordringen in das Bindegewebe fähig werden sollen, nur auf zwei eben so gut in anderem Sinne zu deutende Thatsachen stützen. Die erste ist eben das Vorhandensein des Epithels im Bindegewebe. Man glaubt, es könne dahin nur durch actives Eindringen gelangt sein, übersieht aber dabei, dass eine passive Verlagerung und oin Weiterwachsen des Epithels genau die gleichen Bilder liefern muss. Die zweite ist das lebhafte Proliferationsvermögen des Spithels der Carcinome, das doch nur aus einer biologischen Aenderung erklärt werden könne. Aber diese Erscheinung muss sich ebenso äussern, wenn Zellen, aus dem organischen Zusammenhang getrennt, selbständig sich vermehren. Denn die in allen Zellen unseres Körpers sehlummernde, durch die Einordnung in den normalen Aufbau aber nicht zur Geltung kommende Wachsthumsfähigkeit wird sich frei äussern können, wenn der hemmende Einfluss fortfiel, welchen der Körpertheil, das Organ, der ganze Körper durch die zwischen den einzelnen Zellen bestehenden mechanischen und biologischen Beziehungen, durch die Vertheilung des Gefässsystems, durch das Nervensystem u. s. w. auf die Zellen ausubten. Die isolirten und verlagerten Elemente bethätigen jetzt ihre volle Vermehrungsenergie, die sie den in dem normalen Verband verbleibenden Zellen weit überlegen macht, so dass ihnen von keinem Gewebe dauernder Widerstand entgegengesetzt werden kann. Unter diesen Gesichtspunkten wird es verständlich, dass anch ohne eine Aenderung der biologischen Eigenschaften die Epithelien 
zerstörend vordringen und u. a. auch, wie wir noch besprechen werden, die Epidermis und die Schleimhaut vernichten können, aus der sie selbst hervorgingen.

Wenn nun aber für die Genese des Carcinoms eine passive Metastase von Epithelzellen in das Bindegewebe maassgebend sein soll, so erhebt sich die Frage, wie sie denn zu Stande kommt und ob man sie anatomisch nachweisen kann. Damit komme ich denn zu der von mir in meiner ersten Arbeit beschriebenen bindegewebigen Wucherung, welche durch ihr Vordringen in das Epithel dessen einzelne Zellen abtrennt und so isolirt. Durch diesen Prozess kommt jene zur Carcinomentwickelung führende Metastase zu Stande. Jedoch will ich es auch als möglich hinstellen, dass in seltenen Fällen eine mechanische, traumatische Absprengung in ähnlicher Weise wirken kann.

Ich habe in meiner ersten Mittheilung die anatomischen Beobachtungen, auf die ich mich stütze, ausführlich zur Darstellung gebracht. Theils um sie zu ergänzen, theils um die gegen sie erhobenen Einwände zu entkräften, gehe ich sie nun nochmals einzeln durch.

1. Die Stelle, an welcher ein Carcinom in Entwickelung begriffen ist, springt regelmässig über die Umgebung vor. Die Erscheinung beruht auf progressiven Prozessen im Bindegewebe, welches theils nur lebhafte "zellige Infiltration", theils und zwar gewöhnlich eine lebhafte Neubildung von Zellen und Gefässen zeigt. So entsteht eine subepitheliale Schicht zellreichen Bindegewebes.

2. Durch diese Wucherungsprozesse wird das Epithel gehoben, aber nicht ohne dabei selbst auch Veränderungen zu zeigen. In der Haut verlängern sich nehmlich die Papillen und dementsprechend auch die epithelialen Zapfen, bezw. Leisten. So entsteht also zunächst, strenge genommen, nur eine locale Hypertrophie ohne irgend welche für Carcinom sprechende Eigenthümlichkeiten. Häufig ist bei Hautcarcinomen die Oberfläche dieser Erhebungen papillär, zottig gebaut. Bei beginnenden Schleimhautcarcinomen finden wir analoge Prozesse. Das wuchernde Bindegewebe führt zu einer erheblichen, über das Doppelte und Dreifache hinausgehenden Verlängerung der Drüsen. Es entstehen mehr oder weniger deutlich ausgeprägte Schleim- 
hantpolypen, deren Uebergang in Carcinom bekandtheh vicht selten ist.

Die Bindegewebswucherung ist als eine entzindliche aurzulassen. Es ist aber bekannt, eine wie grosse Rolle chronische Reizzustände bei der Genese der Krebse spielen.

Man könnte nun vielleicht daran denken, dass die Verlängerung der epidermoidalen Zapfen und der Schleimhautdrüsen bereits der Ausdruck eines carcinomatösen Tiefenwachsthums soi, welches in das zuähohst allein gewucherte Bindegewebe erfolgte. Es lässt sich aber leicht zeigen, dass diese Vorstellung irrig ist. Erstens reichen nehmlich die tiefsten Theile der Zapfen nicht über die untere normale Grenze der Epidermis himaus, halten sich vielmehr zu einem grossen Theile genau auf dieser Höhe. Zweitens bieten die bindegewebigen Papillen durchaus das Aussehen von normalen und nur verlüngerten, indem nehmlich die Gefässe in gewohnter Weise central in ihnen nach aufwärts ziehen. Das wäre nicht denkbar, wenn sich zunächst eine Schicht Granulationsgewebe bildete, die doch diese Gefässanordnung nicht zeigen würde und wenn daun in sie das Epithel eindränge. Drittens sind die Zellen in den Zapfen, besonders in den dünnen, sehr lang und schmal, als wären sie, wie ich es ja thatsächlich annehme, durch das Bindegewebe gedehnt. Viertens steht die Verlängerung der Leisten nicht ohne Analogie da. Denn bei den verschiedenartigsten subepithelialen Wucherungszuständen hat man nicht selten Gelegenheit, genau die gleichen Verhältnisse zu sehen. So halte ich es auch nicht für erwiesen, dass irgend welche der als typisch beschriebenen, in das Bindegewebe vordringenden Wucherungen des Epithels aus activem Hineinwachsen derselben entstehen, bin vielmehr der Ansicht, dass auch hier das Emporwachsen des Bindegewebes das Maassgebende ist. Völlig unzweifelhaft ist das bei Geschwülsten der Haut, bei pigmentfreien und pigmentirten Hautwarzen, bei Sarcomen u. s. w. Hier kann man ausserordentlich lange, schmale, vielgestaltige Epithelzüge in dem Geschwulstgewebe nach abwärts ziehen sehen, aber auch nicht tiefer als bis zur normalen unteren Grenze der Epidermisleisten. Solchen Geschwülsten gegenüber wird man wohl nicht an ein actives $\mathrm{Abwärtswuchern} \mathrm{des} \mathrm{Epithels} \mathrm{denken} \mathrm{können.}$ 
3. An die bisher beschriebenen Vorgänge schliesst sich. nun das principiell wichtigste Vordringen des Bindegewebes in das Epithel an. Man sieht die auseinandergedrängten und isolirten Epithelien in das zellreiche Bindegewebe eingelagert. Hauser meint nun aber, das sei nicht die Folge davon, dass das Bindegewebe in die Epidermis hineinwüchse, sondern die Epithelzellen drängen ihrerseits einzeln zwischen die Bindegewebszellen vor. Er giebt also die Richtigkeit der von mir gegebenen Abbildungen zu, deutet sie aber anders. Für mich ergiebt sich daraus die Nothwendigkeit, meine Anschauungen noch einmal zu begründen. Folgende Punkte sprechen für meine Ansicht:

a) Das Eindringen von Zellen bindegewebiger Abkunft in das Epithel ist auch anderweitig bekannt. Lymphkörperchen und Chromatophoren zeigen eine solche Einwanderung.

b) Die oben unter 1 und 2 beschriebenen Anfangsstadien der Krebsentwickelung lassen active Vorgänge fast ausschliesslich am Bindegewebe, am Epithel aber nur so weit erkennen, als natürlich zur Bildung der längeren Zapfen zahlreichere Zellen erforderlich sind, und als dementsprechend eine Vermehrung derselben stattgefunden haben muss. Es ist daher nicht wahrscheinlich, dass nun plötzlich auch das Epithel stärkere active, zu einem Einwachsen in das Bindegewebe führende Proliferation zeigen sollte, während andererseits der umgekehrte Vorgang leicht verständlich ist.

c) An den in das Bindegewebe metastasirten oder in Meta. stasen begriffenen Epithelien bemerkt man nicht nur keine lebhafteren Vermehrungsprozesse als in der übrigen Epidermis, sondern man sieht vielmehr nicht selten, wie ich es auch damals abgebildet habe, an manchen von ihnen Degenerationserscheinungen, die sich in einer homogenen Umwandlung bei Verlust des Kernes äussern und wohl dadurch bedingt sind, dass die abgetrennten Epithelien nicht immer gleich die erforderlichen Existenzbedingungen finden.

d) Besonders beweisend und meiner Meinung uach völlig entscheidend sind die Verhältnisse des von mir beschriebenen beginnenden Magencarcinoms. Ich habe gezeigt, dass die Drüsen in manchen Abschnitten der hypertrophischen Mucosa in einzelne 
Theile zerlegt werden und dass gerade nur unter solehen Stellen die Durchbrechung der Muscularis mucosae beginnt. Dass hier von einem activen Hineindringen des Drüsenepithels in das Bindegewebe nicht die Rede sein kann, geht dentlich genug aus den histologischen Bildern hervor, von denen freilich die meinem orsten Aufsatz beigegebenen Figuren deshalb nur eine nicht völlig ausreichende Vorstellung geben, weil sie, insbesondere die bei starker Vergrösserung gezeichnete, za kleine Abschnitte des Präparates umfassen. Umfangreichere Theile bei genügender Vergrösserung wiederzugeben, verbietet sich aber durch den wverhältnissmässig grossen Umfang, den solche Abbildungen haben müssten. Es scheint mir aber völlig unmöglich, gegenüber den Verhältnissen, bei denen die Struktur der Drüsen völlig aufgehoben wurde und ihre Epithelien einzeln oder gruppenweise durch und in das Bindegewebe versprengt wurden, von einer primären Epithelwucherung reden zu wollen. Es sind ja uberhaupt keine Drüsen mehr da, die im Sinne Hauser's eine primäre krebsige Metamorphose zeigen könnten. Wären freilich die Befande nur an einer einzigen Stelle vorhanden, so könnte man an einen zufälligen, secundären Vorgang denken, aber sie wiederholen sich in allen Schnitten an zahlreichen Orten in der Hauptsache immer in der gleichen Weise. Es kann sich daher nur um typische Verhältnisse handeln. Uebrigens habe ich dieselben Erscheinungen auch, und zwar ebenfalls fleckweise in den Randtheilen eines grösseren Magencarcinoms angetroffen und deute sie hier in gleicher Weise als die Einleitung zur krebsigen Epithelwucherung. Indessen sind solche Objecte weniger beweisend als die ersten Anfangsstadien der Geschwulstentwickelung, wie sie in dem beginnenden Magencarcinom vorlagen.

e) Maassgebend sind endlich auch die oben bereits besprochenen allgemeinen Gründe. Die Annahme eines primären Eindringens einzelner Epithelzellen setzt eine Aenderung des Zellcharakters voraus, für die wir keine Anhaltspunlte haben, die uns daher völlig unverständlich ist. Was soll das Epithel veranlassen, nun mit einem Male in einer der normalen entgegengesetzten Richtung zu wachsen? Für diese Vorstellung bedürfen wir einer eigenen Hypothese, für meine Auffassung ist 
das nicht nöthig, da wir bei ihr vielmehr mit anatomisch nachweisbaren und leicht begreiflichen Momenten rechnen.

Dabei will ich nicht leugnen, dass schon sehr bald ein gegenseitiges Durchwachsen von Bindegewebe und Epithel stattfindet, wie ich auch in meiner ersten Arbeit hervorgehoben habe. Hauser hat geglaubt, darin eine Annäherung an seinen Standpunkt finden zu sollen. Allein ich gebe damit nur zu, dass die isolirt im Bindegewebe liegenden Zellen gleich nach der Isolirung ihre auch in der Norm bethätigte Vermehrung fortsetzen können und sich dann in das Bindegewebe vorschieben müssen. Es scheint mir nun, als wäre meine Darstellung dahin aufgefasst worden, dass die isolirten Zellen nun auch gleich in grössere Entfernung von dem Epidermiszapfen gerathen müssten, um ihre weitere Wucherung bethätigen zu können. Das halte ich aber nicht für nöthig. Jede Epithelzelle, die von den anderen getrennt wurde, mag sie auch Anfangs in ihrer nächsten Nähe liegen bleiben, muss ihre bis dahin einseitige Wachsthumsrichtung ändern. Früher schob sie sich nach anfwärts, wurde gleichsam von den äbrigen dorthin gezogen. Jetzt ist sie selbstständig und bei fortdauernder Vermehrung werden ihre Abkömmlinge sich in der Richtung des geringsten Widerstandes vorschieben. So beginnt also das Wachsthum in das Bindegewebe schon fast sofort nach der Abtrennung der Zellen und insofern tritt eine gegenseitige Durchwachsung von Epithel und Bindegewebe sehr früh ein. Unter allen Umständen aber ist die durch das Eindringen des Bindegewebes bedingte Lösung der Epithelzellen aus dem organischen Zusammenhang der maassgebende Vorgang für die Histogenese des Carcinoms.

Nun sagt aber endlich Hauser, die beschriebene Einlagerung der Zellen in das Bindegewebe käme nicht nur bei beginnenden Carcinomen vor, sie sei vielmehr an der Grenze der schon umfangreichen Cylinderzellenkrebse ebenso gut anzutreffen. Ich weiss das sehr wohl und weiss ebenso, dass Hauser dies in seiner Monographie beschrieben hat. Aber ich lege darauf für die Genese des Carcinoms keinen Werth und habe es deshalb damals unerörtert gelassen. Denn wie das Epithel wächst, wenn es einmal im Bindegewebe liegt, ist für den ersten Anfang 
des Carcinoms, auf den es alloin ankommt, ohne Bedeutung. Wir baben lediglich zu entscheiden, auf welche Weise denn das Epithel an die abnorme Stelle, d. h. in das Bindegewebe hinein gelangt. Ist das aber einmal geschehen, so sind die nun folgenden Vorgänge leicht verständlich.

Hier wendet nun aber Hauser ein, dass die von mir besohriebenen Wucherungszustände des Bindegewebes im Anfang der Krebsbildung nicht immer vorhanden seien. Seine jüngsten Carcinome zeigen indessen bereits verzweigte drüsenähnliche Gebilde in der Submucosa, also nicht mehr die ersten Stadien des Beginns, wie sie in dem von mir untersuchten Carcinom gegeben waren, dem ersten, welches in einem so frühen Stadium zur Beobachtung gelangte. Er beruft sich aber besonders auf die Bilder, die er im Rande von weiter vorgeschrittenen Carcinomen gefunden hat und aus denen er schliessen zu müssen glaubt, dass die dort befindlichen Drüsen sich krebsig umwandelten and die Muscularis durchbrächen, ohne zellige Infiltration des Bindegewebes oder eine sonstige für meine Auffassung sprechende Veränderung.

Hauser verweist dabei auf seine früheren Abbildungen und giebt zwei weitere Figuren, aus denen die krebsige Metamorphose von Drüsen hervorgehen soll.

Ich gebe nun gern zu, dass die Ausführungen Hauser's sehr bestechend sind und dass sie auf denjenigen, der die Entm wickelung des Carcinoms unter den bisher maassgebenden Gesichtspunkten betrachtet, gewiss Eindruck machen. Wenn sie wirklich so gedeutet werden müssten, wie es Hauser thut, so würden sich meine Vorstellungen über die Histogenese des Krebses auch füir die ersten Anfangsstadien nur schwer aufrecht erhalten lassen. Aber so liegt die Sache nicht. Die Gebilde, die Hauser in den dem Carcinom benachbarten Schleimhaut. theilen aus einer krebsigen Metamorphose von Drüsen hervorgehen lässt, entstehen durch Hineinwachsen des bereits gebildeten Tumors in die Mucosa. Ich muss diese wichtigen Verhältnisse eingehender besprechen.

Die Vergrösserung eines zunächst kleinen Carcinoms würde wach Hauser zum grossen Theile dadurch vor sich gehen, dass am Rande immer neue Drüsen krebsig würden und in die Tiefe wucherten. Aber diese Auffassung kann ich nicht theilen. 
Das Breitenwachsthum von Oberflächencarcinomen erfolgt nehmlich in den meisten Fällen nicht dadurch, dass die am Rande angrenzenden, noch normalen Epithelien nun ebenfalls, aber auch dann nur in dem von mir erörterten Sinne, carcinomatöse Wucherung zeigen. So etwas kommt gewöhnlich nur vor, so lange der Krebs noch sehr klein und noch nicht in dem ganzen primär erkrankten Gebiet, sondern etwa nur in den mittleren Abschnitten desselben zur Entwickelung gelangt ist. Verhältnissmässig selten findet sich dieser Modus der Vergrösserung des Tumors noch in den späteren Stadien, wie z. B, in dem oben erwähnten Magenkrebs.

Weitaus am häufigsten schreitet das Carcinom dadurch weiter, dass es allseitig oder nur in einzelnen Richtungen in die Tiefe vordringend, unter das zunächst noch erhaltene umgebende Epithel wächst und dasselbe auf weite Strecken unterminirt. Dadurch entsteht der wallartig aufgeworfene Rand des ulcerirten Krebses. Das über dem Carcinom befindliche Epithel wird gehoben, gespannt, durch Druck zerstört und so in dem nach aussen fortschreitenden Zerfallsprozess der Oberfläche des Tumors hineinbezogen. Man kann nicht selten in der Umgebung von Hautcarcinomen sehen, wie grosse Krebsalveolen von unten gegen die Epidermis andrängen, dieselbe verdünnen und vollständig zur Nekrose bringen. Auf dieselbe Weise kann ja auch die Epidermis über nicht von ihr ausgehenden Carcinomen, z. B. über solchen der Mamma, vernichtet werden.

Das sind ja nun freilich so allgemein bekannte Dinge, dass eine Erläuterung durch Abbildungen überflüssig ist. Aber darauf scheint bisher weniger geachtet worden zu sein, dass die subepithelialen Krebsalveolen und Stränge sich mit den Leisten der zunächst nicht veränderten normalen Epidermis so vereinigen können, dass es den Anschein gewint, als sei von ihnen ein Tiefenwachsthum ausgegangen. Da diese Verhältnisse in Folge des von Hauser erhobenen Einwandes eine besondere Bedeutung gewinnen, so gehe ich auf sie etwas genauer ein und beschreibe als Beispiel ein Carcinom der Stirnhaut, in welchem die Prozesse besonders deutlich hervortreten. In den mir vorliegenden Schnitten, die 
tangential durch den Rand des etwa $1 \mathrm{~cm}$ im Durchmesser haltenden krebsigen Geschwüres geführt sind, ist mit Ausnahme einer kleinen Stelle in der Mitte, die in den Bereich des krebsigen Defectes fällt, das Oberflächenepithel überall erhalten. Unter ihm in der ganzen Breite der Schnitte ist die krebsige Struktur sehr deutlich entwickelt. Man sieht grosse und kleine Alveolen und besonders netzformig zusammenhängende Züge. Es handelt sich also darum, dass von dem ulcerirten Theile aus das angrenzende Corium auf weite Strecken carcinomatös durchwachsen wurde, bei Erhaltung der Epidermis. Das Krebsepithel unterscheidet sich deutlich von der letzteren. Die Kerne sind sind gleichmässig etwas dunkler gefärbt, als die im normalen Rete befindlichen, also etwas chromatinreicher. Der Zellleib ist dagegen etwas kleiner, so dass die Kerne dichter zusammenliegen, als in der Epidermis. Auf diese Weise lassen sich die beiden Epithelarten, das normale und das carcinomatöse, leicht von einander unterscheiden.

Die Krebsentwickelung kommt nun überall der Epidermis nahe, ist aber gewöhnlich von ihr noch durch einen schmalen bindegewebigen Streifen getrennt. An einzelnen Stellen aber und zwar besonders dort, wo die Epidermis längere Leisten aufweist, treten Krebsalveolen und Oberflächenepithel in directe innige Berührung, die theils nur wenig ausgedehnt, theils umfangreicher ist. Figur 1 giebt eine Stelle wieder, an welcher ein Krebszapfen mit einer Epidermisleiste zusammenstősst und seiner äusseren Form nach als directe Fortsetzung derselben ersoheint. Die Grenze beider ist aber an den oben genannten Merkmalen leicht zu erkennen. Eine grössere, daneben gelegene Krebsalveole kommt einem anderen Epidermiszapfen nur nahe, ohne ihn zu berühren. An anderen Stellen liegen lange Alevolen ebenso so langen Leisten in ganzer Ausdehnung an, in einem Abschnitt war im Bereich zweier Papillen das ganze Bindegewebe durch Krebsepithel ersetat, so dass dieses überall an die Epidermis grenzte. Manche Zapfen werden von dem Epithel einer breiten Krebsalveole rings eingehüllt, so dass sie in einer Zellscheide stecken. Dabei können sie die normale scharfe Begrenzung einbüssen und eine zackige Umrandung bekommen. Das liegt daran, dass die Carcinomzellen sich in sie hinein- 
drängen, sie gleichsam annagen, oberflächlich zerstören. Es kann nicht zweifelhaft sein, dass auf diese Weise die Leisten durch den Krebs nach und nach vernichtet werden, dass also ein Epithel das andere zu Grunde richtet. Dasselbe ist möglich, wenn eine kleinere Rrebsalveole an Epidermis anstösst und sich gleichsam in sie hineinfrisst. Alle diese Befunde sind so klar, dass über das gegenseitige Verhältniss von Epidermis und Krebs kein Zweifel bestehen kann. Nicht immer mögen die Bilder so leicht zu deuten sein, aber im Grossen und Ganzen bereiten sie keine Schwierigkeiten. Ich begnüge mich daher mit der Anführung des einen Beispiels und wende mich nun zu den schwieriger zu beurtheilenden Cylinderzellenkrebsen des Darmkanals. Ihre Beziehung zu der angrenzenden Schleimhaut ist eine verschiedene. Entweder stösst hier normale Schleimhaut senkrecht abfallend oder uberhängend an, so dass unveränderte Drüsen am Rande scharf abschneiden, oder die Mucosa ist auf kürzere oder längere Strecken in einer Weise verändert, wie wir es bei hyperplastischen Zuständen, bei Polypen sehen. Die Drüsen sind verlängert, gewunden, geschlängelt, ausgebuchtet, verzweigt, unregelmässig erweitert, die ganze Drüsenschicht ist erheblich verdickt. Man ist leicht geneigt, in diesen Veränderungen die Anfänge der Carcinomentwickelung in dem Sinne zu sehen, dass die Drüsen in einer krebsigen Umwandlung begriffen seien. Indessen müsste man dann auch die Adenome, oder wenigstens viele von ihnen, als Krebse ansprechen, denn auch in ihnen finden sich zahlreiche Abnormitäten der Drüsen. Ich bin aber im Gegentheil der Meinung, dass es keine Veränderung einer in ihrer Continuität erhaltenen Drüse giebt, welche als carcinomatös aufgefasst werden müsste. Ein Carcinom ist nur dann vorhanden, wenn das Epithel in schrankenloser Weise die normalen Grenzen überschreitet. Es würde also in der Darmwand vorhanden sein, wenn nach der gewöhnlichen Auffassung die Drüsen durch continuirliches Wachsthum die Muscularis mucosae durchbrächen. Aber das kommt meiner Meinung nach nicht vor.

Als Einleitung zur Carcinomentwickelung betrachte ich freilich jene hyperplastischen Schleimhautveränderungen auch, aber nur insofern, als an sie sich eventuell die von mir be- 
schriebene Zerlegung der Drïsen wit ihren Folgezustinden anachliesst.

Ausser diesem doppelten Verhalten, also ausser den normalen und den hyperplastischen Zuständen, kann nun der Geschwulstrand selbst schon krebsige Veränderungen dadurch zeigen, dass das bereits gebildete Carcinom auch in ihm und in die weitere Schleimhaut vordringt.

Von dem ulcerirten Theil aus wächst nehmlich das Carcinom sowoh nach abwärts in die Muscularis, wie seitlich anter die angrenzenden, nicht oder nur in der angedeuteten Weiseveränderten Schleimhautbezirkevor. Diesewerden durch die unter ihnen sich entwickelnde Neubildung gehoben usd nach aufwärts gedrängt. Dadurch entsteht der prominirende Rand des Krebses. Ferner aber bemerkt man, dass das Carcinom nicht nur in die Tiefe und in seitlicher Richtung fortschreitet, sondern dass es auch, nachdem os die normale Schleimhaut unterminirt hat, nach oben gegen dieselbe und damit gegen die in ihr befindlichen Drüsen vordringt, von denen es vielfach auf längere oder kürzere Strecken nur noch durch die Muscularis mucosae getrennt ist. Aber eben wegen dieser anfänglich stets noch, in manchen Fällen davernd vorhandenen, continuirlich erbaltenen, trennenden Muskelschicht sind solche Präparate besonders geeignet zar Demonstration des Breitenwachsthums des Carcinoms.

In einzelnen Fällen kommt nun eine Zerstörung der über dem Krebs liegenden Schleimhaut lediglich auf mechanischem Wege durch den auf die Mucosa oinwirkenden Druck des wachsenden Krebses zu Stande. Mir liegt ein Präparat vor, in welchem der Randwulst eines krebsigen Mastdarmdefectes folgende Verhältnisse zeigt. Die normale Schleimhaut erhebt sich gegen das Geschwür hin allmählich über das unter ibr vordringende Carcinom und flacht sich mehr und mehr ab bis auf etwa ein Drittel der normalen Dicke. Dabei werden die Drüsen zunächst nur schräg gerichtet, schliesslich aber ganz parallel der Oberflächo des Randwulstes. Sie verlieren ihr Lumen, das Protoplasma wird niedriger und nur doppelte Reihen von Kernen lassen auf untergehende Drüsen schliessen. Weiterhin verlieren sich die comprimirten Drüsen allmählich ganz, die Mucosa geht über in eine 
Schicht ohne deutliche Struktur, die sich in die obersten, aus nekrotischen Massen bestehenden Lagen des Geschwürs fortsetzt. Die so veränderte Schleimhaut ist, so lange sie sich noch als solche erkennen lässt, überall in scharfer Linie gegen das andrängende Carcinom begrenzt, wenn sich auch die Muscularis mucosae nicht mehr deutlich abhebt.

In anderer Weise aber wird die Schleimhaut zerstört, wenn das Carcinom nicht nur mechanisch wirkt, sondern bald hier, bald dort, an einzelnen oder vielen Stellen die Muscularis mucosae durchwächst und in die Schleimhat eindringt. Hier tritt es in verschiedene Beziehung zu den Drüsen, wie im Einzelnen genauer besprochen werden muss.

So sehe ich in einem Object in einiger Entfernung von dem scharf abschneidenden Rande des Geschwürs eine Gruppe von Alveolen bis zwischen die untersten Enden der normalen Drüsen vorragen. Eine schlauchförmige Krebsalveole berührt sich mit einem Drüsenfundus. In keinem Schnitt aber findet eine wirkliche Vereinigung statt. Es sind nur drei Schnitte, in denen die Annäherung so weit geht, in den anderen ist die Entfernung wieder grösser. Es handelt sich also um eine kegelförmig nach oben ragende Krebswucherung, die mit ihrer Spitze gerade die Drüsen berührt.

An einer anderen Stelle ist das Verhalten ganz analog. Die Krebsalveolen sind aber grösser, ihre ganze Gruppe ist breiter, die Spitze des Kegels daher stumpfer. Aber auch sie geht bis an die Drüsen heran, ohne irgendwo mit ihnen zasammenzuhängen.

Ein weiteres Carcinom liefert wieder neue Bilder. An mehreren Stellen sind Krebszüge bis in die Schleimhant, an einer bis nahe an die Oberfläche vorgedrungen, so dass die obersten Alveolen etwa noch um ein Fünftel einer Drüsenlänge davon entfernt sind. Das Carcinom bildet hier einen rundlichen Alveolenhaufen, an dem sich nach unten ein zweiter anschliesst, der dann direct zusammenhängt mit den tieferen Krebsmassen. Die oberste Alveolengruppe der Schleimhaut nimmt etwa die Breite von vier Drüsen ein und das Bindegewebe zeigt zellige Infiltration. $W_{0}$ sind nun die früher an dieser Stelle vorhandenen Drüsen? Sie finden sich in comprimirtem Zustande 
seitlich neben und über den Krebsalveolen. Man kann sie zum Theil noch gut orkeanen, aber sie sind zusammengedrückt, entsprechend verschmälert und auf die Seite gedrängt. In anderen Schnitten kann man sie schwer erkennen, schlecht gegen das umgebende zellige Gewebe abgrenzen, in wieder anderen sind sie nur mit Mühe noch als modificirte Drüsen anzusprechen. Auch zwischen den Carcinomalveolen und der Oberfläche der Schleimhaut bemerkt man comprimirte und verdrängte Drüsen. Diese Verhältnisse machen es aber völlig klar, dass die krebsigen Epithelgebilde nicht etwa durch eine atypische Drüsenwucherung entstanden sind, sie lassen vielmehr keine andere als die hervorgehobene Deutung zu.

In einem ferneren Careinom sehe ich die Verdrängung von Drüsen durch Krebsmassen gerade an dem Rande des Geschwürs, (Figur 2). Dieses fällt scharf ab, aber seine Begrenzung ist nicht von normalen Drüsen gebildet, sondern von einem die Fortsetzung der normalen Mucosa darstellenden Gewebe, welches otwa die Breite von 4-5 Drüsen einnimmt, aber typisch carcinomatös gebaut ist. Dieser Gewebsabschnitt könnte, wenn die bisherigen Betrachtungen nicht eine andere Anschauung nahe legten, als eine durch directe Drüsenwucherung carcinomatös umgewandelte Mucosa angesehen werden. Dagegen spricht aber schon der Umstand, dass die Alveolen nicht grösser, sondern eher geringer an Zahl sind, als die auf einem entsprechenden Bezirk vorhandenen Drüsen. Das liesse sich bei einer aus letzteren hervorgehenden Wucherung nicht wohl denken. Man sollte ferner erwarten, dass die krebsige Umwandlung nicht so scharf abschnitte, aber wir sehen neben Krebsalveolen Drüsenschläuche, die keine Spur von carcinomatösen Prozessen darbieten, sondern nur durch die angrenzenden, neugebildeten Massen bei Seite gedrängt werden. Ich leite also auch diese Befunde aus einem Hinaufwachsen des Krebses in die Schleimhant ab. Aber freilich wird dieser Vorgang wohl kaum erst eingetreten sein, als das Geschwür bereits den jetzt vorhandenen Umfang erreicht hatte, sondern schon früher. $Z$ u einer Zeit, als dort, wo jetzt schon das Ulcus vorhanden ist, noch Schleimhaut sich befand, wachs in der oben besprochenen Weise das Carcinom an manchen Stellen, u. a. auch an der hier in Betracht kommenden, nach 
aufwärts in die Mucosa vor und verdrängte die hier befindlichen Drüsen. Als dann das Geschwür sich ausdehnte, gelangte es auch an diesen so veränderten Bezirk, der nun zur Zeit der Exstirpation des Tumors gerade den Rand des Defectes bildete. Gegen diese Auffassung wird man verschiedene, zum Theil auch aus der Figur 2 abzuleitende Einwände erheben.

Man wird sagen, man sehe ja deutlich, dass die neben den Drüsen gelegene Krebsalveole (b) in die Mündung einer normalen Drüse überginge, also doch wohl aus einer Umwandlung der letzteren hervorgegangen sei. Das ist aber eine Täuschung. Bei starker Vergrösserung erkennt man, dass beide Dinge gar nicht zusammengehören, sondern dass zwischen ihnen ein, auch in der Zeichnung angedeutetes, bindegewebiges Septum liegt. Es handelt sich also darum, dass die Carcinomalveole auf der dem Beschauer zugewandten Seite der Drüse nach oben wächst und dass es nun in dem etwas schräg gefallenen Schnitt so aussieht, als sei jener Zusammenhang vorhanden. Ein solcher Irrthum ist auch dann möglich, wenn ein Krebsstrang seitlich an eine Drüse heranwächst (Fig. 3). Die Berührung kann so innig sein, dass eine genetische Beziehung zu bestehen scheint. Genauere Betrachtung mit starker Vergrösserung giebt auch hier die Aufklärung, die durch gleichzeitige Untersuchung der zunächst angrenzenden Schnitte erleichtert wird. Man könnte nun aber hervorheben, dass diese Deutung zwar vielleicht in einzelnen Fällen, aber durchaus nicht immer möglich sei, dass man vielmehr nicht selten jenen Zusammenhang mit voller Bestimmtheit wahrnehmen könne. Dem gegenüber muss ich zunächst zugeben, dass in der That mit Lumen versehene Krebsschläuche sich bis zur Oberfläche der Schleimhaut erstrecken und hier ausmünden können. Aber man darf daraus nicht schliessen, dass es sich um primär carcinomatös umgewandelte Brüsen handele. Es sind vielmehr zwei andere Erklärungen dieser Befunde möglich. Erstens kann der Abschnitt, durch welchen die Ausmündung auf die Oberfläche stattfindet, wirklich einer Drüsenmündung entsprechen. Dann ist aber die übrige Drüse nicht krebsig umgewandelt, sondern durch eine von unten heraufgedrungene Krebswucherung ersetzt und zerstört. Eben so gut wie die Carcinomschläuche in das Bindegewebe hineindringen und die Drüsen 
nur bei Seite drängen könnea, ist auch ein Mineinwachsen in eine Drüse möglich. Ja, es dürfte das sich ganz passiv verhaltende Epithel der letzteren geringeren Widerstand bieten, als das Bindegowebe. Es wird daher von dem Krebsepithel leicht zerstört werden. Auch bei der Epidermis sahen wir ja, dass ihre Zellen durch das Carcinom vernichtet werden und wenn es a uffallend erscheinen könnte, dass das Drüsenepithel so völlig verdrängt würde, so verweise ioh auf die weiter unten folgenden Schilderungen des Verhaltens des Cylinderepithels zur Epidermis. Dort werden wir sehen, dass letztere durch ersteres völlig aufgezehrt werden kann. Nun wird man aber Beweise für meine Auffassung verlangen. Indessen könnte ich zunächst eben so gut Beweise für die primäre krebsige Degeneration fordern. Denn sie ist lediglich aus der Thatsache abgeleitet, dass man eine Brüse sich in einen Krebsschlauch fortsetzen sieht. Für meine Darstellung aber kann ich ausserdem zwei Punkte anführen. Binmal kommt jene, von Hauser angenommene carcinomatöse Vetamorphose nur vor, weun das unter der Schleimhaut gelegene Bindegewebe schon von Krebs durchwachsen ist. Man sollte aber doch erwarten, dass sie auch einmal so früh angetroffen würde, dass bei intacter Mucosa eben erst der Durehbruch durch die Muscularis mucosae erfolgte. Zweitens ist das Aufwärtswachsen und das Hineindringen des Carcinoms in die Mucosa mit voller Bestimmtheit nachgewiesen. Die nothwendigen Grundlagen für die angenommene Zerstörung der Drüsen durch heraufgedrungene Krebsschläuche sind also gegeben und so ist meine Juffassung die besser begründete. Eine Illustration dazu liefert aber auch eine Abbildung Hauser's in seiner Monographie. In Fig. 15 ist nehmlich nach seiner Meinung eine Drüse gezeichnet, die in grösster Ausdehnung krebsig umgewandelt ist. Dabei sollen aber nahe der Mündung, mitten zwischen Krebsepithelien, noch mehrere Cylinderzellen der carcinomatösen Metamorphose nicht verfallen sein. Ich halte es für näherliegend anzunehmen, dass sie von dem von unten heraufgewachsenen Krebs nicht zerstört wurden.

Die zweite Erklärung für die freie Ausmündung von Krebsschläuchen liegt nun in einem Heraufwachsen derselben im Bindegewebe bis zur Oberfläche und in einer auf letztere erfol- 
genden Eröffnung des Lumens durch Zerfall der obersten Epithelzellen. Für diesen Vorgang liefert die Fig. 2 ebenfalls eine Erläuterung. $A n$ dem in das Geschwür abfallenden Theile des Randes (bei d) sieht man neben einander zwei unzweifelhafte Krebsalveolen frei ausmünden. Sie etwa als modificirte Drüsen aufzufassen, ist deshalb ganz ausgeschlossen, weil sie dicht an der Muscularis mucosae und horizontal liegen. Das Bild lehrt also, wie leicht man zu falschen Schlüssen kommen kann, wenn man jeden auf die Oberfläche ausmündenden Epithelschlauch ohne Weiteres als umgewandelte Drüse auffassen wollte. Das nunmehr zu schildernde Präparat liefert. u. a. auch noch eine werthvolle Ergänzung zu dem Emporwachsen der Krebsschläuche bis zur Oberfläche.

Es handelt sich um ein Rectumcarcinom, welches bis zum Anusring herabreichte und dadurch in Beziehung zur Epidermis trat. Da auf diese $W$ eise $z$ wei ganz verschiedene, leicht zu unterscheidende Epithelarten in Betracht kommen, so müssen sich ihre gegenseitigen Beziehungen ganz besonders leicht untersuchen lassen. In der That gestatten die hier vorliegenden Verhältnisse gar keine Meinungsverschiedenheiten und verlangen daher eine genauere Besprechung.

Der Rand des Geschwürs bildet einen stark vorspringenden, von Epidermis bekleideten und von dem Cylinderzellenkrebs durchwachsenen Wulst, dessen Oberfläche in den mikroskopischen Schnitten eine Ausdehnung von $1 \frac{1}{2}-2 \mathrm{~cm}$ hat. Auf diese Strecke ist also die Epidermis von dem Carcinom unterminirt, von dessen ulcerirter Fläche an dem einen Ende der Schnitte ein kleiner Theil zu sehen ist. An ihn schliesst sich, nur wenig höher im Niveau gelegen, eine etwa $1 \mathrm{~mm}$ lange Strecke wohlerhaltener, dann ein eben so langer Abschnitt in Nekrose begriffener, kernloser oder mit zerbröckelten Kernen versehener, dann wieder bis zum anderen Ende des Schnittes unveränderte Epidermis an. Auf eine Entfernung von etwa $1 \mathrm{~cm}$ von dem Geschwür, dringen die Krebsalveolen bis nahe an die Epidermis vor, während sie im übrigen Schnitte etwas weiter davon entfernt bleiben. Dort sind nun die Bilder sehr verschieden. Ein Theil der Alveolen ist noch von der Epidermis durch eine schmale Bindegewebszone getrennt, ein anderer Theil reicht bis 
dicht an sie, bezw. bis an einzelne Zapfen heran, wieder ein anderer geht eine innige Verbindung mit ihr ein, wobei die Berührungsflächen bald breit, bald schmaler sind. Es kommt auch vor, dass die Kuppe einer Papille ganz durch eine Krebsalveole ausgefüllt wird (Fig. $5 a, c$ ). Die Grenze zwischen den beiderseitigen Epithelien ist natürlich wegen der verschiedenen Form und wegen der dunkleren Färbung der Cylinderzellen leicht zu erkennen, aber nicht immer eine völlig scharfe. Zieht man lediglich die Lagerungsbeziehungen in Betracht, so kann eine Carcinomalveole, die mit einem Zapfen verschmilzt, sehr wohl a's die directe Fortsetzung desselben erseheinen. Das wahre Verhältniss ist ja aber ohne Weiteres einleuchtend. Die weitere Wucherung des Krebses hat aber nun zu interessanten Verdrängungs- und Zerstörungserscheinungen an der Epidermis geführt. Letztere wird bald hier, bald dort angenagt und in grösserem Jmfange durch Cylinderzellen ersetzt. Sehr deutlich geht das aus Fig. 4 hervor. Ein breiter Epidermiszapfen, der sich rechts oben aus dem Oberflächenepithel entwickelt, ist durch eindringendes Cylinderepithel von unten her zu einem grossen Theil zerstört und gleichsam auggehöhlt. Das Cylinderepithel bildet eine unregelmässige, noch ungefähr schlauchförmige Figur und ist an Stelle völlig verschwundener Epidermis getreten. Nur körnige und fädige Massen im Lumen könoten als Epithelreste gedeutet werden, aber auch sie sind, wie aus den analogen Substanzen in den übrigen Krebsschläuchen entnommen werden darf, wohl als Produkte der Cylinderzellen unter Beimengung einiger Leukocyten aufufassen. Die Grenze der Krebsepithelien gegen die erhaltene und unveränderte Epidermis ist meist ganz scharf. Das Bild illustrirt also die völlige Aufsaugung der Epidermis durch die Cylinderzellen und damit auch die oben besprochene Möglichkeit, dass eine Drüse durch Cylinderzellen eines Krebsschlauches ersetzt werden kann ohne Spuren zu hinterlassen. Noch ein anderer Punkt der früheren Besprechungen findet in dem jetzt vorliegenden Object eine weitere Erläuterung. Fig. 5, in welcher rechts und links je eine Krebsalveole eine Papille ausfüllt, während links eine darunter gelegene Alveole einen Zapfen nach oben verdrängt, zeigt in der Mitte eine Unterbrechung der Zellschichten der Epidermis, währead 
die Hornschicht erhalten ist. Jene Lücke ist erzengt von einem Krebsschlauch (d), der nach aufwärts wachsend die Epidermis bis zur Hornchicht durchbrochen und zerstört hat. Wenn auch die letztere noch beseitigt wäre, so würde der Schlauch, dessen Cylinderzellen sich beiderseits an die den Rand der Unterbrechung bildende Epidermis anlegen, frei an der Oberfläche ausmünden und so eine Drüse, bezw. einen aus ihr hervorgegangenen Krebsstrang vortäuschen. Was aber hier möglich ist, kann selbstverständlich auch in den oben besprochenen Fällen im Bereich der Darmschleimhaut geschehen.

So ergänzt also dieses letzte Präparat die früheren Erörterungen in ausserordentlich klarer, unzweidentiger Weise. Wenn ich daher das über das Wachsthum der Schleimhautearcinome Gesagte noch einmal überblicke, so komme ich zu dem Schluss, dass die von Hauser erhobenen Einwände nicht stichhaltig sind. Alle seine Darstellungen und Abbildungen lassen sich auf die von mir angegebene Weise, also durch Emporwachsen des unter der Schleimhaut vorgedrungenen Carcinoms gegen diese und in sie hinein, erklären. Sie sind also nicht geeignet, meine oben noch einmal begründete und vertheidigte Anschauung über die Histogenese des Carcinoms irgendwie zu erschüttern. Es würde zu weit führen, wollte ich an der Hand der Literatur zeigen, dass auch die in früheren Arbeiten auf ein Tiefenwachsthum des angrenzenden Epithels bezogenen Bilder in der angegebenen Weise zu erklären sind. Erinnert sei aber daran, dass auch bei Virchow's Lehre von der bindegewebigen Genese des Carcinoms sehr gewöhnlich eine Vereinigung mit dem darüber liegenden Epithel zu Stande kommen müsste und dass Koester (Die Entwickelung der Carcinome. 1869) für das nach seiner damaligen Meinung aus den Lymphgefässendothelien entstehende Carcinom besonders auf diese Erscheinung hingewiesen hat. Hervorgehoben sei ferner, dass meine Darstellung auch für die Drüsencarcinome gilt, in denen die vordringenden Krebsalveolen auf normale Theile stossen. So entsteht die Täuschung, als seien die Krebsstränge aus modificirten Alveolen hervorgewachsen. Es ist daher nach Allem unmöglich, an ausgebildeten Carcinomen die Histogenese festzustellen. 
Zum Schluss noch einige, Bemerkungen über die A tiologie der subepithelialen, hyperplastischen Zustände des Bindegewebes. Es ist nicht zu bezweifeln, dass die Wucherung durch die versehiedensten chronischen Reizwirkungen, die 5ekanntlich bei der Entstehung der Carcinome eine grosse Rollo spielen, hervorgorufen werden kann. So gewinnen wir eine gemeinsame Grundlage für die Aetiologie der krobsigen Neubildungen. Ich darf darauf verzichten, diese Gesichtspunkte weiter $z a$ verfolgen. Nur auf einen Punkt möchte ich noch mit einigen Worten eingehen, nehmlich auf die Beziehungen zwischen $\mathrm{Car}$ cinom und Tuberculose. In einem, diesem Gegenstand gewidmeten Aufsatz ${ }^{1}$ ) habe ich es als möglich bezeichnet, dass die Tuberkelbacillen in einem Theil der Fälle dasjenige Agens darstellen könnten, welches die als maassgebend für die Carcinomgenese angesprochenen subepithelialen Granulationsprozesse hervorriefen. Ich stuitzte mich dabei auf die Beobachtungen von gleichzeitigem Vorkommen der beiden Erkrankungen, auf das Hervorgehen von Krebsen aus Lupus und auf eine Reihe von mir erhobener histologischer Befunde. Ich fand in dem Bindegewebe von Carcinomen knötchenförmige zellige Gebilde, die in den wesentlichsten Punkten mit Tuberkeln übereinstimmten und deren Riesenzellen ich nicht als Fremdkörperriesenzellen deuten konnte. Freilich gelang es mir nicht, Bacillen nachzuweisen, aber ich konnte aus Mangel an Material nur wenige Zellen darauf untersuchen. Demgemäss sprach ich mich auch nicht mit voller Bestimmtheit aus, musste aber per exclusionem zu der Auffassung von der tuberculösen Natur der besprochenen riesenzellenhaltigen Neubildungen gelangen.

Meine Darstellung ist nun aber in zwei auf Anregung von Iubarsch entstandenen Arbeiten angefochten worden und zwar in der von Clement ${ }^{2}$, „über seltenere Arten der Combination von Krebs und Tuberculose" und in der von Krïckmaun ${ }^{3}$ ) „über Fremdkörpertuberculose und Fremdkörperriesenzellen“. Ersterer beschreibt mehrere Fälle von Carcinom, in denen die regionären Lymphdrüsen tuberculös waren. Dabei ist es mit

1) Münchener med. Wochenschr. 1894. No. 17.

2) Dieses Archiv. Bd. 139. \$. 35.

a) Ebenda. Bd.138. Supplement S. 118. 
Rücksicht auf meine obigen Mittheilungen und die folgenden Auseinandersetzungen von Wichtigkeit, dass Clement die Tuberculose mehrfach aus der Gegenwart typischer Riesenzellen erschliesst, ohne Bacillen nachgewiesen zu haben. Er hält nun die Tuberculose insofern für den secundären Prozess, als sie bis dahin latent war, aber durch die wahrscheinlich chemische Wirkung des Krebses manifest wurde. Gewiss kann man sich den Zusammenhang so denken, allein gezwungen ist man dazu nicht. Ich könnte mir auch hier sehr wohl vorstellen, dass die Tuberculose, die immerhin längere Zeit latent verlaufen sein mag, durch ihre Localisation in der Haut, der Schleimhaut, der Drüse die Grundlage für die Genese des Carcinoms abgab.

Clement giebt nun zu, dass meine Anschaung von der Entwickelung des Krebses in vieler Hinsicht plausibel and anziehend sei, hält aber ihre Durchführbarkeit für fraglich. Insbesondere sei Lubarsch der Ansicht, dass die Carcinome des Uterus und der Mamma dagegen sprächen. Es ist mir nicht recht verständlich, worauf dieser Satz sich stützen lässt, da doch über beginnende Krebse der beiden Organe bisher gar keine Untersuchungen vorliegen. Clement erkennt ferner an, dass meine Angaben über die Tuberculose geeignet seien, die Entstehung des Carcinoms auf lupöser Basis zu erklären. Meine Deutung der von mir erhobenen Befunde hält er aber für falsch. Er weist auf die Arbeit Krückmann's hin, der nachgewiesen habe, dass auch Fremdkörperriesenzellen in Carcinomen vorkommen. Mit diesen soll ich in meinen Fällen, vielleicht mit Ausuahme eines einzigen, die Riesenzellen verwechselt haben. Krückmann führt denn auch zwei Beobachtungen von Carcinom mit vielkernigen Zellen gegen meine Auffassung an. Ich bedaure die Behauptungen von Clement, da sie den Vorwurf oberflächlicher Untersuchang einschliessen. Er versucht freilich eine Milderung desselben, indem er hervorhebt, dass die Fremdkörper nicht so grob und dass sie nicht in den ZeIlen zu liegen brauchten. Aber auch so hätte er mir wohl zutrauen können, dass ich die Verwechselung zu vermeiden im Stande wäre, zumal ich in meiner Arbeit selbst zwei Fälle von Carcinom anführe, in denen ich die vielkernigen Elemente als Fremdkörperriesen- 
zellen anspreche. Immerbin balte ich es nicht für überitussig die Gründe meiner Darstellung nun noch einmal zu erörtern.

Krückmann's Befunde sind von deu meinigen ganz verschieden. Er findet in einem Carcinom des unteren Augenlides theils zwischen den Epithelien, theils im Bindegewebe, aber scharf gegen dasselbe abgesetzt, Riesenzellen, welche verhornte Epithelien als Fremdkörper einschliessen. In einem Carcinom des Oesophagus beschreibt er ferner Riesenzellen zwischen den Epithelzellen. Hätte Krückmann bei Abfassung seiner Arbeit die Untersuchungen Dünschmann's') schon gekannt, der ebenfalls in Carcinomen in directer Beziehung zum Epithel viele Riesenzellen beobachtete, so würde er sie auch gegen meine Auffassung verwerthet haben. Aber alle diese Befunde unterscheiden sich von den meinigen sehr wesentlich. Ausnehmen möchte ich nur, wie ich es auch damals schon als möglich hinstellte, den ersten meiner Fälle, da hier die Riesenzellen mit Epithelien zusammenliegen. In den übrigen Fällen dagegen war ¡ch aus folgenden Gründen zu dem Schlusse gezwungen, dass Fremakörperriesenzellen auszuschliessen seien.

1. Die Riesenzellen liegen ohne Beziehung zam Epithel mitten im Bindegewebe, zum Theil weit von jenem entfernt. Das ist bei Krückmann nicht der Fall.

2. Die Riesenzellen bilden meist das Centrum bindegewebiger, zelliger, zum Theil reticulär gebauter, knötchenförmiger Bezirke, die in der Hauptsache Tuberkeln entsprechen. Das ist ebenfalls bei Krückmann nicht der Fall.

3. Die Riesenzellen schliessen keine Fremdkörper, keine verhornten Epithelien, keine anderen Zellen ein. Auch darin liegt ein Unterschied gegenüber den Präparaten Kr ückmann's.

Ich habe also zunächst keine Veranlassung, meine damalige Deutung aufzugeben, will mich aber gern belehren lassen, wenn mir nachgewiesen wird, dass ausser den durch Fremdkörper veranlassten und ausser don tuberculösen noch andere Riesenzellen im Carcinom und zwar im zellig infiltrirten Bindegewebe vorkommen können. Im Uebrigen ist es für meine Vorstelluug ₹on der Histogenese des Carcinoms ohne Bedeutung, ob in einer

1) Journal of Pathology. Bd. III. Hft. !. 
grösseren oder nur in einer kleinen Zahl der Fälle jene bindegewebigen Wucherungsprozesse durch Tuberkelbacillen bedingt sind. Welche ätiologische Momente auch immer in Betracht kommen mögen, stets ist das Grundlegende die durch das Vordringen der Bindegewebselemente bedingte Trennung der Epithelien aus ihrem organischen Zusammenhang und ihre Verlagerung in das Bindegewebe.

\section{Erklärung der Abbildungen. Tafel $\mathrm{V}$.}

Fig. 1. Subepidermoidale Krebsentwickelung in einem Carcinom der Stirn. Die Krebsalveolen wachsen gegen die Epidermis, eine von ihnen ist mit einem Epidermiszapfen vereinigt und bildet scheinbar die directe Fortsetzung desselben.

Fig. 2. Rand eines ulcerirten Carcinoms des Dickdarms. Bei a normale Drüse, daneben eine durch einen Krebsscblauch (b) verdrängte und comprimirte. Der Krebsstrang steht scheinbar in Zusammenhang mit einer über ihm befindlichen Drüsenmündung (c). Bei $d$ münden zwei Krebsalveolen frei am Rande aus, so dass sie Drüsen vortäuschen können.

Fig. 3. Unteres Ende einer Drüse (a), im Rande eines Rectumcarcinoms. Von der einen Seite drängt eine Krebsalveole (b) gegen sie an, comprimirt sie und steht so scheinbar mit ibr in directem Zusammenbang.

Fig. 4. Aus einem unter die angrenzende Epidermis vorgedrungenen $\mathrm{Cy}$ linderzellenkrebs des Rectums. Von unten her ist ein Krebsstrang in einen Epidermiszapfen eingedrungen und hat ihn in unregelmässiger Weise zerstört und ausgeböblt.

Fig. 5. Aus demselben Carcinom. Links füllt eine Krebsalveole (a) eine Papille aus, darunter hat eine zweite (b) einen Epidermiszapfen theilweise verdrängt. Rechts gleicbfalls eine Alveole (c) in einer Papille. In der Mitte ist ein Krebsschlauch (d) in die Epidermis eingewuchert und bat sie bis auf die Hornschicbt zerstört, so dass er nach deren Entfernung frei ausmünden würde. 